\title{
Low-grade glioma subtypes revealed
}

\section{ce \\ We were able to clini- cally define molecular subgroups of pLGG}

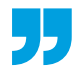

Low-grade gliomas (LGGs) are the most common brain tumours in children and can arise from glial cells, neuronal cells or both. This heterogeneity is also reflected in the diverse range of anatomical locations of these tumours within the CNS and in treatment outcomes, especially if surgical resection is either incomplete or not feasible. In an attempt to address this heterogeneity, Cynthia Hawkins and colleagues conducted an integrated clinical and molecular analysis of $>1,000$ paediatric LGGs (pLGGs).

Hawkins explains: "Over the past decade, our group and others have identified alterations impacting the RAS/MAPK pathway, most commonly BRAF, as the near universal driver of the disease. However, patient outcomes and responses to therapy are highly variable so a better idea of who needs aggressive therapy and who can forgo therapy altogether is desperately needed." In this study, investigators adopted a tiered approach to molecular interrogation, beginning with a series of clinically validated molecular diagnostic assays, with sequencing-based approaches required in only a third of samples.

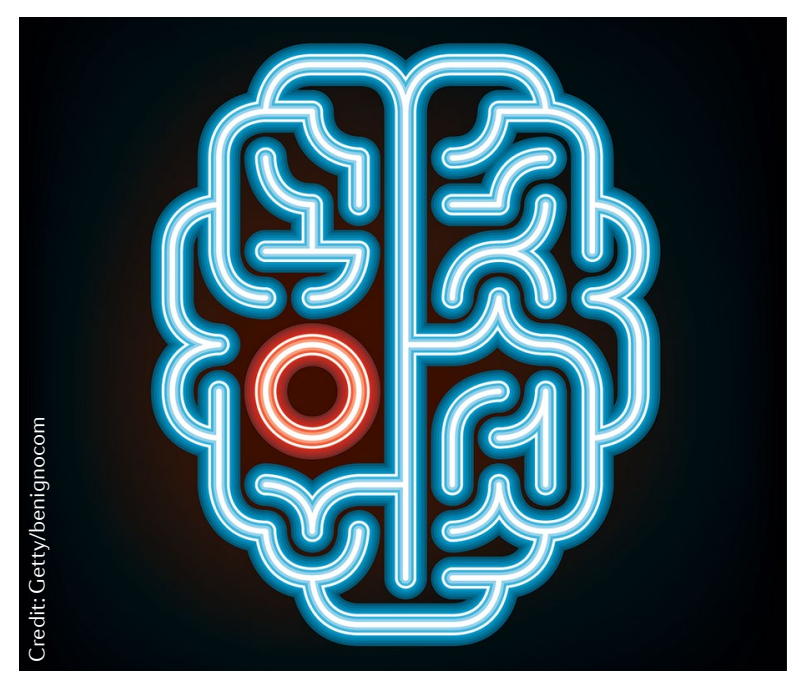

Overall, median age at diagnosis was 7.6 years, and, at a median overall survival (OS) follow-up duration of 15.9 years, only $7.5 \%$ of patients had died. Disease progression was observed in $33 \%$ of patients, with a median time to progression of 2.3 years (median progression-free survival (PFS) follow-up duration 5.9 years).

Among NF1-driven pLGGs $(n=133 ; 14 \%)$, which arise from germline mutations in the gene encoding neurofibromin, investigators identified a high-risk subgroup $(n=25)$ with primary tumour locations outside of the optic pathway. Of these, five tumours were found to harbour other known driver alterations, and these patients had inferior outcomes.

In an analysis of all samples with sufficient material available $(n=477)$, $84 \%$ of pLGGs were found to harbour at least one driver alteration. One of three alterations: KIAA1549-BRAF (35\%); BRAF $F^{\mathrm{V} 600 \mathrm{E}}(17 \%)$; and germline NF1 mutations (17\%) was found in $68 \%$ of samples analysed. Other alterations included those involving FGFR1/2 (6.1\%), other receptor tyrosine kinases (RTKs) (3.4\%) or genes with no direct effects on MAPK signalling (4.6\%). Notably, gene-set enrichment analyses revealed no significant differences in activation sequences between a subset of pLGGs and other tumours, regardless of the presence or absence of targetable alterations affecting MAPK signalling, implying responsiveness to MAPK targeted therapies, even in the absence of a targetable driver alteration.

Remarkably, a significant association was observed between the type of alteration (fusion versus single-nucleotide variant (SNV)) and outcome. Fewer deaths were observed among patients with fusiondriven $\mathrm{pLGGs}$ versus $\mathrm{SNV}$-driven pLGGs (7\% versus $24 \%$; $P<0.0001$ ), reflected in 10-year OS rates of $97.8 \%$ versus $88.1 \%$. Further stratification revealed an association between $H 3 F 3 A^{\mathrm{K} 27 \mathrm{M}}$ and inferior outcomes (median time to progression 0.8 years) in the 12 patients with alterations of this type. Among patients with fusion-driven pLGGs, those involving the fusion of exon 15 of KIAA1549 with exon 9 of BRAF were associated with the worst prognosis (5-year PFS 59\%).

The investigators used these data to define three distinct risk groups: low risk, comprising patients with tumours driven by fusions or NF1 alterations (20-year OS 96\%); intermediate risk, including those with tumours harbouring $B R A F^{\mathrm{V} 600 \mathrm{E}}$ without $C D K N 2 A$ deletions, or SNVs in several other RTKs and $I D H^{\mathrm{R} 132 \mathrm{H}}$ (20-year OS 81\%); and high risk, including those with tumours driven by $H 3 F 3 A^{\mathrm{K} 27 \mathrm{M}}$ or $B R A F^{\mathrm{V} 600 \mathrm{E}}$ with CDKN2A deletion (10-year OS 41\%).

Senior author Uri Tabori summarizes: "In this study we stratified patients with pLGG into three risk categories with clinical recommendations on how to manage each alteration and risk group. This includes a 'watch and wait' and cytotoxic therapy-sparing approach for low-risk tumours and an increasingly aggressive approach for intermediate and high-risk patients".

Hawkins concludes: "We were able to clinically define molecular subgroups of pLGG. This included an analysis of subgroups of the common alterations (KIAA1549-BRAF and $\left.B R A F^{\mathrm{V} 600 \mathrm{E}}\right)$ revealing higher-risk subtypes of these entities that were previously not described".

These finding provide guidance on the prognostication and treatment stratification of patients with pLGGs harbouring specific molecular alterations and might facilitate clinical trials involving selected patient subgroups.

Peter Sidaway

ORIGINAL ARTICLE Ryall, S. et al. Integrated molecular and clinical analysis of 1,000 pediatric low-grade gliomas. Cancer Cell 37, 569-583 (2020) 